\title{
A Synthesis Method of Nonlinear Transformation and Affine Transformation for Constructing NIFS
}

\author{
Shuqun Liu, Guodong Zhu ${ }^{\mathrm{a},{ }^{*}}$ \\ School of Computer and Community, Lanzhou University of Technology, Lanzhou 730050, China \\ ahz_zhugd@sina.com
}

Keywords: Fractal; Nonlinear iterated function systems (NIFS); Deterministic iteration algorithm; Random iteration algorithm; Attractors.

\begin{abstract}
In order to construct a large number of diverse and innovative fractal graphics, an arbitrary nonlinear transformation of $V(x, y)$ is introduced into the classical iterated function systems to change the construction form of nonlinear iterated function systems (NIFS). The deterministic algorithm and random iteration algorithm are used to draw the fractal graphics. The summarization of relevant transformation rules are obtained according to the generation of NIFS attractors. The experimental results show that the combination of nonlinear transformation and affine transformation enriches the transformation types of iterated function systems, and a simple and effective approach is provided for the drawing of NIFS fractal graphics.
\end{abstract}

\section{Introduction}

In 1985, M.F. Barnsley presented a theory of iterated function systems (IFS) [1]. IFS is an important fractal theory and is an important method to generated fractal graphics. IFS has important theoretical significance and has practical application value in the fields of natural landscape simulation and image compression. The traditional IFS has some limitations to construct fractal graphics. The NIFS modeling, however, is extremely flexible and expressive, and the constructed fractal graphics using NIFS are more natural and abundant.

The method of constructing NIFS by mapping $\chi \leftarrow a_{0} \chi^{2}+a_{1} \chi+a_{2}$ with polynomial in the real number range is proposed in [2]. But it has not been used for the drawing of fractal graphics. The Fractal Flame algorithm proposed in [3], the algorithm is applied to the fractal software Apophysis, used to the generation of the fractal graphics of colorful and varied. The bilinear transformation is used for quadrangle on the basis of compression transform in [4]. The method provides a new idea for the drawing of the fractal graphics. Under the plural transform perspectives, the literature [5] discusses the definition of linear plural transform $Z=a Z+t_{i}$, the iteration convergence theorems of plural transform, and the properties of fractal graphics, and a new patterning method has been proposed. Based on the linear complex mapping, a type of nonlinear complex mapping families $f(Z)=a Z^{2}+t_{i}$ are studied in [6] to form the condition of an iterated function systems, and the relationship of the initial point selection and the generated attractor is discussed. Combine the linear affine transformation and square root function, the literature [7] provides the method of drawing fractal graphics when the initiator is regular polygons or circles. The representation form based on polynomial transformation in [8] is applied to draw the fractal graphics, increasing the diversity of fractal attractors. However, the transformation involved is limited to quadratic transformation.

The construction method based on IFS fractal curve is proposed in [9]. By choosing the appropriate parameters for a given family of contraction maps, and combining the method of constructing a regular polygon by a linear iterated function and the method of constructing a curve based on a complex planar nonlinear iterated function $z \longleftarrow \sqrt{e^{i \theta} z}$, the construction method extends the mapping of IFS and NIFS construction fractal graphics to the plural form. The Literature [10] constructs NIFS using two single parameter complex polynomials and proposes the method of establishing a set of parameters for iteration mapping, to construct fractal graphics on the plane. In the literature $[11,12]$, the method of constructing nonlinear IFS by non-analytic complex mapping $f(z)=e^{i \frac{\pi}{2}} z^{n}+c$ is studied. And, the novel structural fractal mountains and the fractal graphics with $D_{n+1}, Z_{n+1}$ symmetrical 
characteristics are generated. The method of constructing nonlinear IFS by the negative integer-power complex mapping family $f(z)=z^{-n}+c$ is also studied.

In view of the shortcoming of drawing fractal graphics with traditional IFS, in this paper, we research the NIFS attractor structure model according to the construction model of the fractal flame algorithm in [3], and the related experiments simulations and analysis are carried out. The specific methods are as follows: Based on fractal flame model and the proposed model, this paper introduces the nonlinear transformation (mainly in some classical IFS). By using the combination of affine transformation, and the combination of nonlinear transformation, nonlinear transformation and affine transformation respectively, we compare the generations of NIFS attractors and summarize the experimental rules. The proposed method enriches the theoretical model of NIFS and provides more possibilities. The proposed method is a more powerful method for NIFS modeling control.

\section{Basic Theory}

\subsection{Iterated Function Systems}

Let $\mathrm{X}$ denotes a complete metric space with distance function $\mathrm{d}$ and $W_{n}$ being a mapping from $\mathrm{X}$ into itself.

Definition 1: A iterated function systems is consisted of a compact metric space (X,d), a finite set of compression maps $W_{n}: X \rightarrow X$ and the corresponding compression factors of $\alpha=\max \left\{\alpha_{n} ; n=1,2, \cdots, N\right\}$.

Theorem 1: Let $\left\{X ; W_{n}, n=1,2, \cdots, N\right\}$ be iterated function systems (IFS), with compression factor ' $\alpha$ '. Then the transformation $W_{n}: X \rightarrow X$ is defined as $W(B)=\bigcup_{n=1}^{N} W_{n}(B), \forall B \in H(X)$; It is a compression mapping on the compact metric space $(H(X), h(d))$ with compression factor " $\alpha$, that is

$h(W(A), W(B)) \leq \alpha h(A, B), \forall A, B \in H(X)$.

Then there exists a fixed point set $P \in H(X)$ called the attractor of the IFS, such as $P=W(P)=\bigcup_{n=1}^{N} W_{n}(P)$ and $P=\lim _{n \rightarrow \infty} W^{n}(B), \forall B \in H(X)$.

The form of transformation $P=W(P)=\bigcup_{n=1}^{N} W_{n}(P)$ is:

$W_{n}\left[\begin{array}{l}x \\ y\end{array}\right]=\left[\begin{array}{ll}a_{n} & b_{n} \\ c_{n} & d_{n}\end{array}\right]\left[\begin{array}{l}x \\ y\end{array}\right]+\left[\begin{array}{l}e_{n} \\ f_{n}\end{array}\right]$, that is $W_{n}(x, y)=\left(a_{n} x+b_{n} y+e_{n}, c_{n} x+d_{n} y+f_{n}\right)$,

Where $a_{n} \vee b_{n} \vee c_{n}{ }^{\prime} d_{n} \vee e_{n} f_{n}$ are the coefficients of affine transformation.

Select a symmetrical regular quadrilateral with an edge length of 1 as the initiator, and observe the generation processes of the finite iteration and the sierpinski triangle attractor, as shown in Figure 1.

Table 1. The IFS code of sierpinski triangular

\begin{tabular}{cccccccc}
\hline$W_{n}$ & $a_{n}$ & $b_{n}$ & $c_{n}$ & $d_{n}$ & $e_{n}$ & $f_{n}$ & $p$ \\
\hline$W_{1}$ & 0.5 & 0 & 0 & 0.5 & -0.5 & -0.5 & 0.33 \\
$W_{2}$ & 0.5 & 0 & 0 & 0.5 & 0.5 & -0.5 & 0.33 \\
$W_{3}$ & 0.5 & 0 & 0 & 0.5 & 0 & 0.366 & 0.34 \\
\hline
\end{tabular}

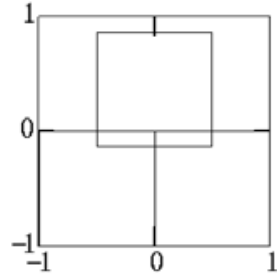

(a)The first iteration

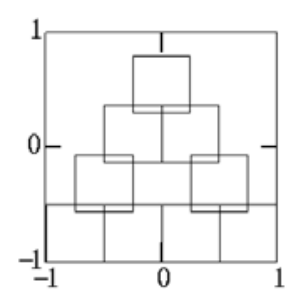

(b)The second iteration

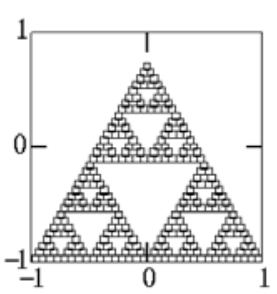

(c)The fifth iteration

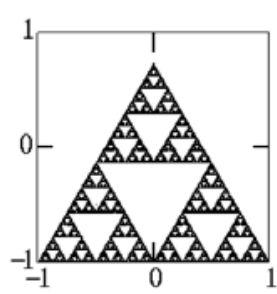

(d)attractor

Figure 1. The generation process of sierpinski triangle attractors 


\subsection{The Fractal Flame}

The fractal flame algorithm mentioned in [3], was created by Scott Draves in 1992. Different from the traditional iterated function systems, the fractal flame algorithm defines a series of nonlinear transformation $_{V_{j}}$. The core idea of the algorithm is random iteration algorithm. To randomly select points $\mathrm{P}$, in each iteration, the algorithm randomly select transformation $V_{i}(x, y)=V_{j}\left(a_{i} x+b_{i} y+c_{i} d_{i} x+e_{i} y+f_{i}\right)$ to get the next point of $\mathrm{P}$ according to the probability. The form of transformation is:

$$
F_{i}(x, y)=\sum_{\mathrm{j}} v_{i j} V_{j}\left(a_{i} x+b_{i} y+c_{i}, d_{i} x+e_{i} y+f_{i}\right) ; i, j=0,1,2, \cdots, N
$$

Where the parameters $v_{i j}$ are the weightings of the nonlinear transformation $V_{j}$, and the sum of the weighting coefficients is $1 . a_{i} \vee b_{i} \vee c_{i}{ }^{\prime} d_{i}{ }^{\prime} e_{i}{ }^{\prime} f_{i}$ are the coefficients of the affine transformations.

\section{NIFS Attractors}

This section mainly introduces a construction model of NIFS, several nonlinear transformation, NIFS generation algorithm, and gives the examples to illustrate the generation process of NIFS attractors.

\subsection{The Construction Method of NIFS}

The transformation of fractal flame is obtained by adding affine transformation based on the nonlinear transformation. In this paper, The method of constructing NIFS is the opposite to the method of constructing fractal flame algorithm, that is, affine transformation is added on the basis of the nonlinear transformation. Then, We obtained the following modeling methods:

$$
\begin{gathered}
\left({ }_{s}(x, y), t(x, y)\right)=\sum_{i} w_{i} V_{i}(x, y) \\
W_{i}(x, y)=\left(a_{\mathrm{i}} s+b_{i} t+c_{i}, d_{i} s+e_{i} t+f_{i}\right) ; i=0,1,2, \cdots, N
\end{gathered}
$$

Where the parameters $w_{i}$ are the weightings of the nonlinear transform $V_{i}(x, y)$. The sum of the weighting coefficients is 1 , and $a_{i} \vee b_{i} \vee c_{i} \vee d_{i} \vee e_{i} \vee f_{i}$ are the coefficients of affine transformations.

\subsection{Nonlinear Transformations}

We selects several primitives' transformation of fractal flame in literature [3], in this paper, as shown in Fig.2:

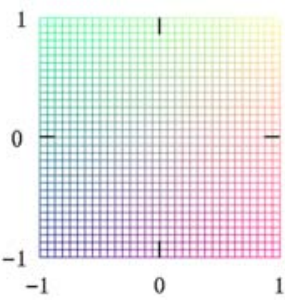

(a)Initial

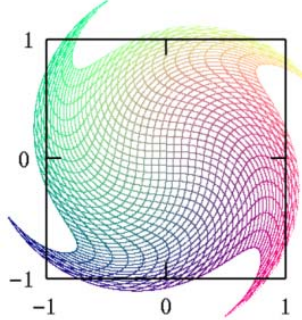

(b)swirl

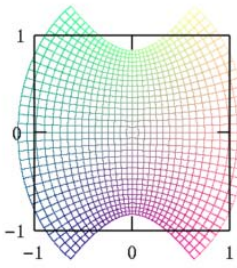

(c) $\sinh$

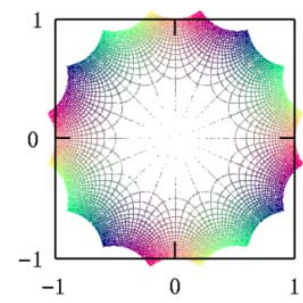

(d)juliaN

The analytic expression is:

Figure 2. Nonlinear transformation

$$
\begin{aligned}
& V_{\text {swirl }}(x, y)=\left(x \sin \left(r^{2}\right)-y \cos \left(r^{2}\right), x \cos \left(r^{2}\right)+y \sin \left(r^{2}\right)\right) \\
& V_{\text {sinh }}(x, y)=(\sinh (x) \cdot \cos (y), \cosh (x) \cdot \sin (y)) \\
& p_{1}=\text { juliaN.power, } p_{2}=\text { juliaN.dist; } \\
& p_{3}=\operatorname{trunc}\left(\left|p_{1}\right| \Psi\right) \\
& \quad t=\left(\varphi+2 \pi p_{3}\right) / p_{1} \\
& V_{\text {julian }}(x, y)=r^{\frac{p_{2}}{p_{1}}} \cdot(\cos t, \sin t)
\end{aligned}
$$

Where $r=\sqrt{x^{2}+y^{2}}, \varphi=\arctan (y / x), p_{1} p_{2}$ is real number, in this paper the parameter of $p_{1}$ is $5, p_{2}$ is $1, \Psi$ is a random variable uniformally distributed on the interval of $[0,1]$.

We mainly consider the following two aspects: 
The first, although the synthesis transformation is still a nonlinear transformation, but due to the type of nonlinear transformation is too numerous, there is no uniform way of setting parameters and control methods. However, selecting some nonlinear transformation that the parameters are fixed, the form of nonlinear transformation is controlled by affine transformation. It can not only extend the expressive ability of the nonlinear transformation, but also simpler and easier to control.

The second, affine transformation are used to synthesize nonlinear transformation, rather than the conversed construction. That is because the style of nonlinear transformation has a foreseeable relationship with the corresponding style after affine transformation, and the constructed NIFS attractors are relative much intuitionist.

\subsection{NIFS Attractors Generation Algorithm}

Although the method proposed in this paper is different from the fractal flame algorithm, but we still approximate the NIFS attractors using random iteration algorithms.

The implementation of the algorithm is described below:

Choose any one of the initial points $P\left(x_{0}, y_{0}\right)$, according to the mapping sequence and the corresponding probability distribution. Random selection transform and plots a new coordinate point. According to this rule, a new coordinate point is continuously generated, until it reaches the maximum number of iterations, gradually approaching NIFS attractors.

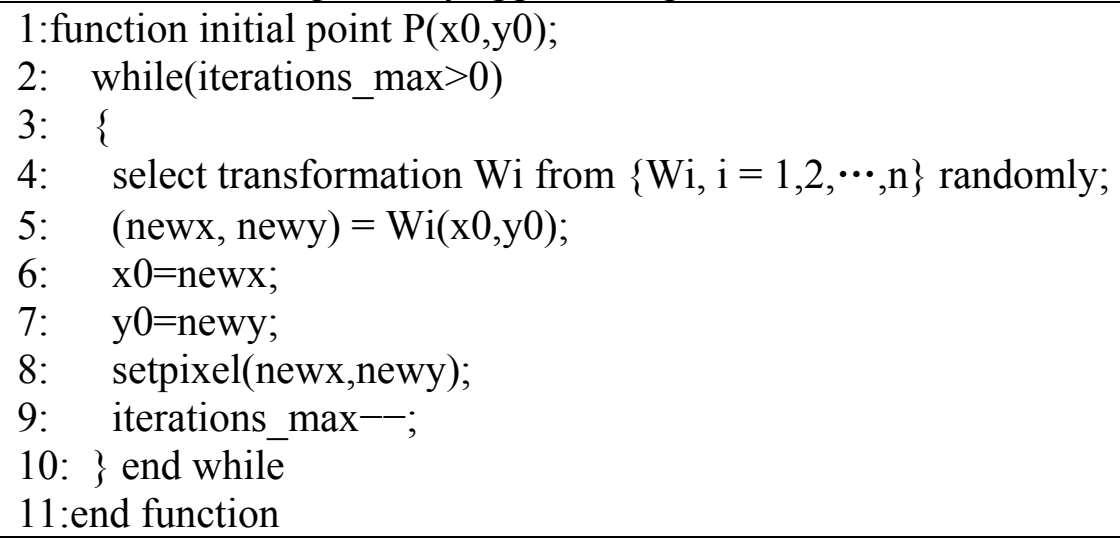

\subsection{Finite Times Iteration and NIFS Attractors Gereration}

Using the method proposed in this paper, we add swirl transformation to sierpinski triangle, and the iteration process NIFS attractors is shown in Figure 3.

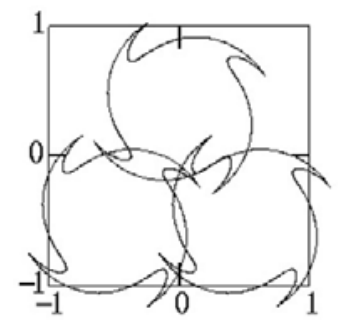

(a)The first iteration

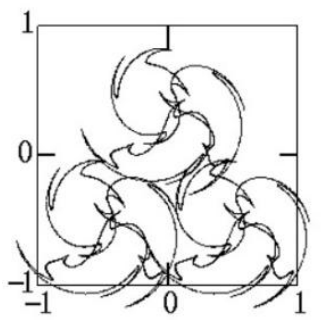

(b)The second iteration

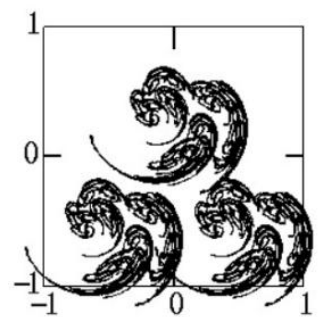

(c)The fifth iteration

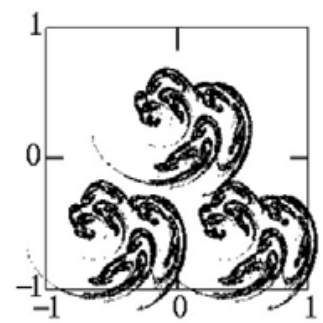

(d)attractor

Figure 3. The generation process of NIFS attractors

\section{Nifs Attractors Generation Effect and Comparison}

In this section, we select the above swirl, sinh, and juliaN transformation. A nonlinear iterated function system is constructed on the classical IFS. Using random random iteration algorithm to approximate NIFS attractors, and observe the effect of fractal graphics generated, as shown in Figure 4. 


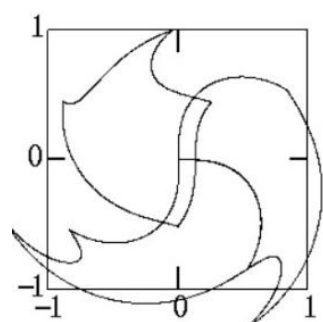

(a-1) Collage schematic

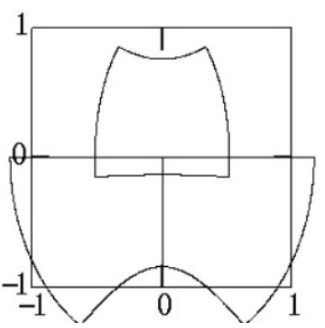

(b-1) Collage schematic

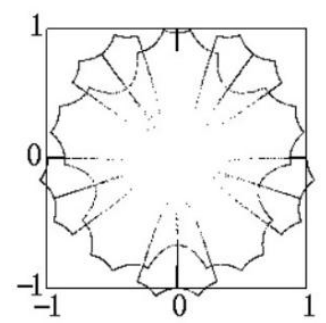

(c-1) Collage schematic

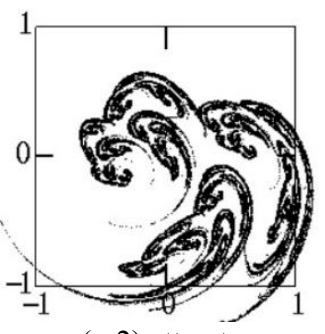

(a-2) attractor

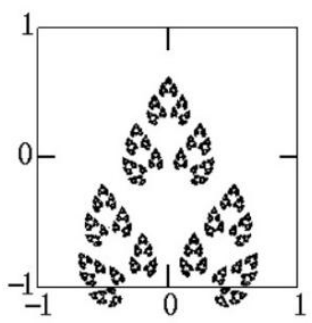

(b-2) attractor

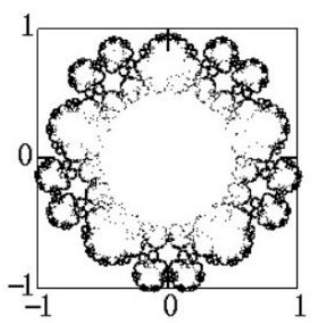

(c-2) attractor

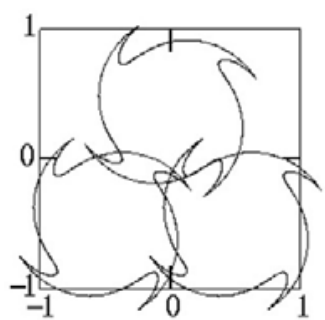

(a-3) Collage schematic

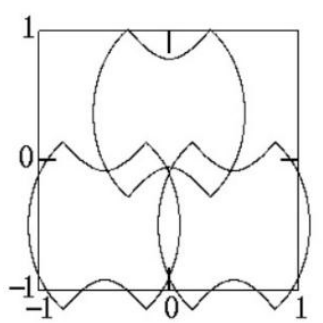

(b-3) Collage schematic

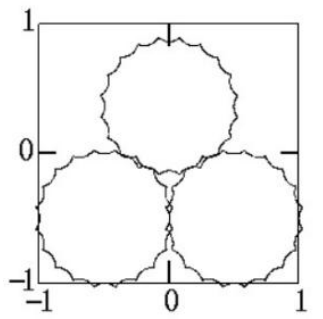

(c-3) Collage schematic

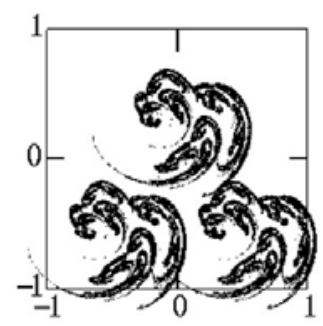

(a-4) attractor

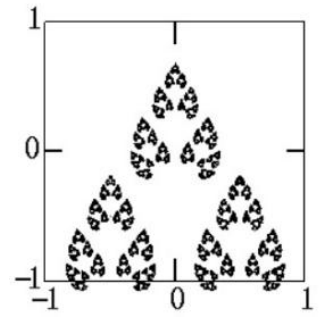

(b-4) attractor

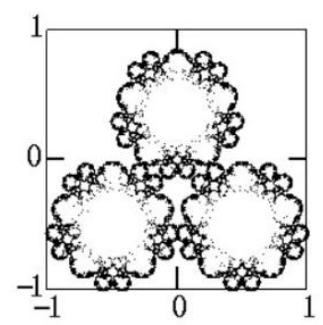

(c-4) attractor

Figure 4. Fractal graphics generated by the fractal flame method and the suggested method

By comparing the above NIFS attractor's renderings, we can see that the classic sierpinski triangle chosen above has a symmetrical structure. Respectively, using fractal flame method and the method of this paper, the structure of their NIFS attractors has some association. That is, the latter is similar in structure with the former, and the number of similar structures is related to the number of affine transformation. Based on this, using the transformation method proposed by this paper on the classical IFS $\left(Z_{n}, D_{n}\right.$ symmetry, symmetry about the origin, axisymmetry), more fractal graphics with symmetrical structure can be constructed.

The fractal graphics with $Z_{n} 、 D_{n}$ symmetrical structure and its effect picture of adding transformation. Their IFS codes of affine transformation are shown in Table 2 and Table 3 . The IFS attractors of $D_{4} 、 Z_{5}$ symmetrical structure and the effect diagram of adding transformation are shown in Figure 5.

Table 2. The IFS code of $D_{4}$ Symmetrical

\begin{tabular}{lccccccc}
\hline$W_{n}$ & $a_{n}$ & $b_{n}$ & $c_{n}$ & $d_{n}$ & $e_{n}$ & $f_{n}$ & $p$ \\
\hline$W_{1}$ & -0.25 & -0.30 & 0.30 & -0.34 & 0.50 & 0.50 & 0.125 \\
$W_{2}$ & -0.30 & 0.34 & -0.25 & -0.30 & -0.50 & 0.50 & 0.125 \\
$W_{3}$ & 0.25 & 0.30 & -0.30 & 0.34 & -0.50 & -0.50 & 0.125 \\
$W_{4}$ & 0.30 & -0.34 & 0.25 & 0.30 & 0.50 & -0.50 & 0.125 \\
$W_{5}$ & -0.25 & -0.30 & -0.30 & 0.34 & 0.50 & -0.50 & 0.125 \\
$W_{6}$ & 0.30 & -0.34 & -0.25 & -0.30 & 0.50 & 0.50 & 0.125 \\
$W_{7}$ & 0.25 & 0.30 & 0.30 & -0.34 & -0.50 & 0.50 & 0.125 \\
$W_{8}$ & -0.30 & 0.34 & 0.25 & 0.30 & -0.50 & -0.50 & 0.125 \\
\hline
\end{tabular}


Table 3 . The IFS code of $Z_{5}$ Symmetrical

\begin{tabular}{cccccccc}
\hline$W_{n}$ & $a_{n}$ & $b_{n}$ & $c_{n}$ & $d_{n}$ & $e_{n}$ & $f_{n}$ & $p$ \\
\hline$W_{1}$ & 0.5000 & 0 & 0 & 0.500 & 0.5000 & 0 & 0.20 \\
$W_{2}$ & 0.1545 & -0.4755 & 0.4755 & 0.1545 & 0.1545 & 0.4755 & 0.20 \\
$W_{3}$ & -0.4045 & -0.2939 & 0.2939 & -0.4045 & -0.4045 & 0.2939 & 0.20 \\
$W_{4}$ & -0.4045 & 0.2939 & -0.2939 & -0.4045 & -0.4045 & -0.2939 & 0.20 \\
$W_{5}$ & 0.1545 & 0.4755 & -0.4755 & 0.1545 & 0.1545 & -0.4755 & 0.20 \\
\hline
\end{tabular}

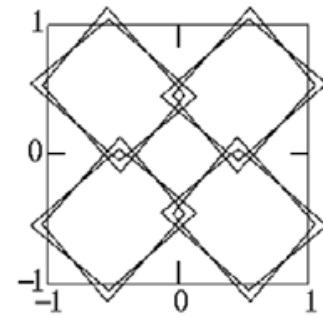

$D_{4}$ Collage schematic

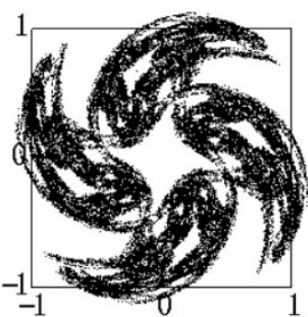

Fg 1(swirl)

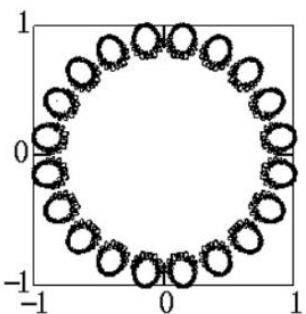

Fg 5(julia)

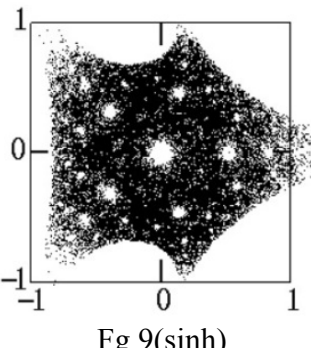

Fg 9(sinh)

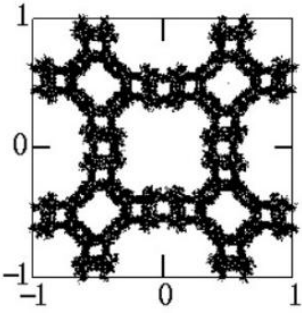

(a)attractor

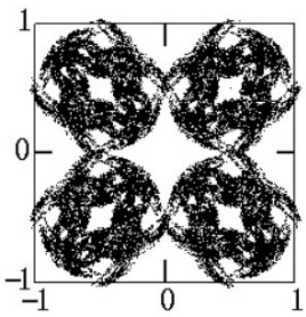

Fg 2(swirl)

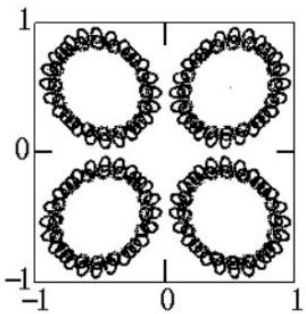

Fg 6(julia)

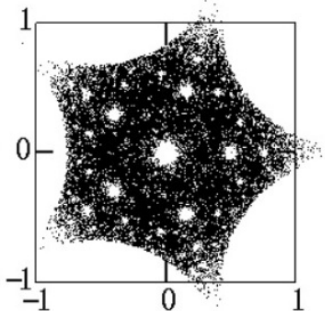

Fg 10(sinh)

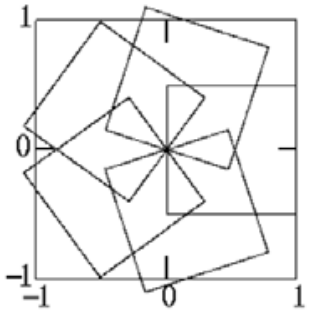

$Z_{5}$ Collage schematic

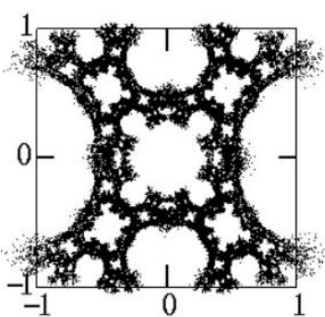

Fg 3(sinh)

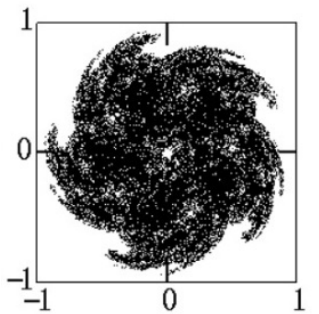

Fg 7(swirl)

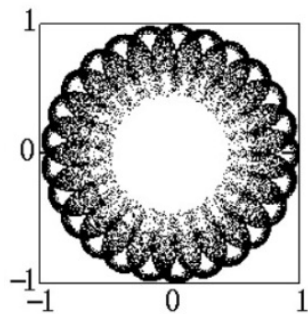

Fg 11(julia)

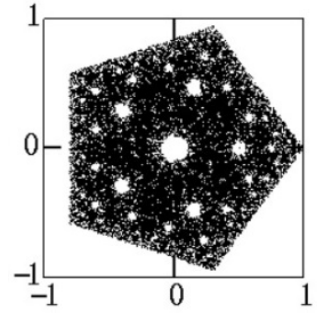

(b)attractor

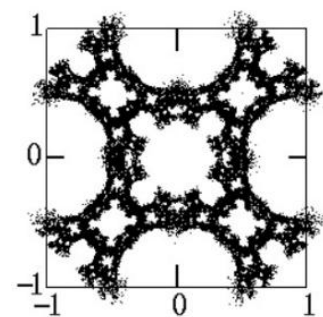

Fg 4(sinh)

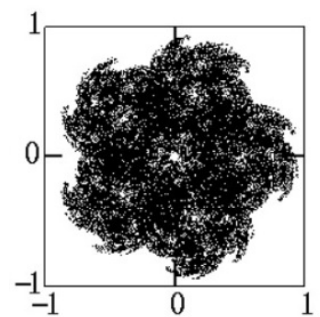

Fg 8(swirl)

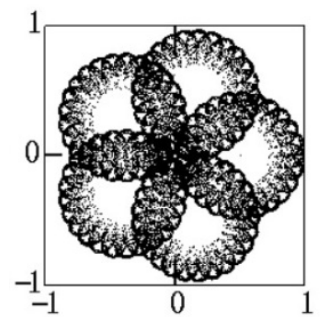

Fg 12(julia)

Figure 5. Fractal graphics with symmetrical structure

Where, The fractal graphics Fg 1(Fg 7), Fg 3(Fg 9) and Fg 5 are obtained by using fractal flame. The fractal graphics Fg 2(Fg 8), Fg 4(Fg 10) and Fg 6(Fg 12) are obtained by using the method of this paper. By comparing the above effects diagram, we can draw the following conclusions: for a symmetrical IFS, a large number of fractal graphics with symmetric features and novel structures can be constructed by using the combination of nonlinear transformation and affine transformation; The NIFS attractors constructed in this paper is related to the value n, such as, For Fg 2, Fg 4 and Fg 6, it is shown there have four similar structures. In fact, there have eight similar structures, that is because the IFS with $D_{4}$ symmetric has eight affine transformation with similar structure. For Fg 8, Fg 10 and 
Fg 12, they all have five similar structures, because the $Z_{5}$ symmetric IFS has five affine transformations of similar structure, and overlap occurs between the structures.

In the following part, this paper selects a more general (asymmetric) IFS to add nonlinear transformation, the IFS codes of affine transformation is shown in Table 4, the generation effect of NIFS attractors is shown in Figure 6.

Table 4 the IFS code of orchid

\begin{tabular}{cccccccc}
\hline$W_{n}$ & $a_{n}$ & $b_{n}$ & $c_{n}$ & $d_{n}$ & $e_{n}$ & $f_{n}$ & $p$ \\
\hline$W_{1}$ & 0.5 & 0.25 & 0.25 & -0.5 & 0 & 0 & 0.5 \\
$W_{2}$ & 0.75 & -0.25 & 0.25 & 0.75 & 0.75 & 0 & 0.5 \\
\hline
\end{tabular}

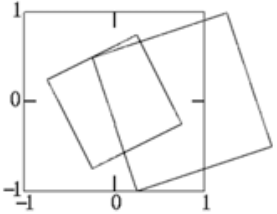

(a) Collage schematic

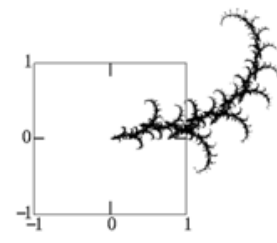

(b)attractor

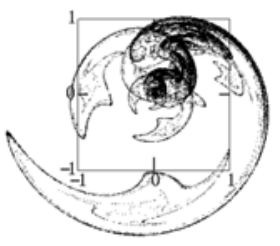

(c)fractal flame

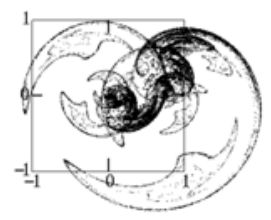

(d)the suggested method

Figure 6. Asymmetric IFS add nonlinear transformation

Where, the picture $\mathrm{c}$ and $\mathrm{d}$ of Figure 6 are obtained by adding swirl transformation with fractal flame algorithm and the method of this paper respectively. By comparing the Figure 6, we can find that it can generate strange attractor with the suggested method. And by using affine transformation, the attractors generated by the two methods can be converted to each other.

\section{Conclusion and Prospect}

In this paper, we put forward a method of constructing NIFS based on classical IFS and nonlinear transformation. Different from the fractal flame algorithm, it uses affine transformations based on nonlinear transformations. So it ensures that the type of transformation constructed has a central character. And then, using random iteration algorithm to draw NIFS attractor, nonlinear transformation are added to the IFS with symmetrical characteristics and asymmetric characteristics. By experiments and comparisons, further explore the relationship between them. The experimental results indicate that the method can be applied to the drawing of fractal graphics. It can not only form a variety of fractal graphics with novel shapes, but also provides a new perspective for the study of NIFS architecture, and it greatly extends the expression ability of transformation. In the future work, the rendering method of NIFS, the law of nonlinear transformation and the NIFS architecture were worth further studying.

\section{References}

[1]. Barnsley M F, S Demko. Iterated function systems and the global construction of fractals[C]. Proceedings of the Royal Society of London, A. Mathematical Physical \& Engineering Sciences, 1985, 399(1817), pp. 243-275.

[2]. E.R. Vrscay, D. Weil. "Missing moment" and perturbative methods for polynomial iterated function systems [J]. Physica D Nonlinear Phenomena, 1991, 50(3), pp. 478-492.

[3]. Scott Draves, Erik Reckase. The Fractal Flame Algorithm [EB/OL]. http://flam3.com/flame draves.pdf. 2008.

[4]. E Gröller. Modeling and rendering of nonlinear iterated function systems [J]. Computers \& Graphics, 1994, 18(5), pp. 739-748. 
[5]. FAN Cui-fang, LIN Yi-xia. Application of Plural Transform $Z=a Z+t_{i}$ in Fractal Composition [J]. Journal of Shanghai University, 2003, 9(2):139-144.

[6]. ZHANG Xi-zhe, WANG Zheng-xuan, PANG Yun-jie. The condition of complex mapping family $f(Z)=a Z^{2}+t_{i}$ to become an IFS [J]. Journal of Engineering Graphics. 2005, 26 (2), pp. 114-118.

[7]. Philip Van Loocke. Non-linear iterated function systems and the creation of fractal patterns over regular polygons [J]. Computers \& Graphics, 2009, 33(6), pp. 698-704.

[8]. LIU Shu-qun, LIU Luo-Sheng. Iterated function system based on polynomial transformation [J]. Journal Lanzhou University of Technology, 2011, 37(1), pp. 81-85.

[9]. Dong Jie, CHEN Ning, WANG Feng-ying. Research on Fractal Construction Method Based on Complex Transformation $z \leftarrow \sqrt{e^{i \theta} z}[\mathrm{~J}]$. Journal of Chinese Computer Systems, 2016, 37(6), pp. 1344-1347.

[10]. Chen Ning, Feng Dongdong. Nonlinear IFS from complex quadratic polynomials with single complex parameter [J]. Journal of Computer-Aided Design \& Computer Graphics, 2016, 28(2), pp. 255-259.

[11]. Chen Ning, Wu Xiaochen, Li Ming. Research on Parameters of Construction of IFSs from Non-Analytical Complex Mappings [J]. Journal of Computer-Aided Design \& Computer Graphics, 2017(7), pp. 1267-1274.

[12]. Chen Ning, Li Ming, Wu Xiao-chen. Construction of Fractals from the Analytical Complex Mapping Family with Negative Inerger Powers [J]. Journal of Chinese Computer Systems, 2017, 38(9), pp. 2166-2170. 